\title{
PRIMER REGISTRO DE PROBSTMAYRIA TAPIRI (NEMATODA: ATRACTIDAE) EN TAPYIRUS BAIRDII (GILL, 1865) DE LA SIERRA MADRE DEL ESTADO DE CHIAPAS, MÉXICO
}

\author{
Darío M. GÜIRIS ANDRADE1, Nidia M. ROJAS HERNÁNDEZ², \\ Vicente BERovides Álvarez ${ }^{2}$, Epigmenio CRUZ ALDÁN ${ }^{3}$, \\ Carlos Chávez Hernández ${ }^{3}$, Jorge A. Moguel ACuÑa ${ }^{3}$, \\ María E. Pérez Escobar ${ }^{1}$, M. Gabriela Palacios Mendoza ${ }^{3}$ \\ ${ }_{1}^{1}$ Facultad de Medicina Veterinaria y Zootecnia. Universidad Autónoma de Chiapas. Departamento de \\ Ciencias Médico Veterinarias. Jefe de Operaciones Policlínica y Diagnóstico Veterinario (particular), \\ Blvd. Ángel Albino Corzo 635-B. C.P. 29040. Tuxtla Gutiérrez, Chiapas, MÉXICO. \\ dmguiris@hotmail.com. \\ 2 Facultad de Biología. Coordinación de posgrado. Depto. Microbiología. Universidad de la Habana, \\ CUBA \\ 3 Oficina de Investigación. Zoológico Regional "Miguel Álvarez del Toro", Instituto de Historia \\ Natural y Ecología del Gobierno del Estado de Chiapas (IHNE). Calzada a Cerro Hueco s/n Colonia \\ El Zapotal Tuxtla Gutiérrez Chiapas, MÉXICO.
}

Güiris Andrade, D. M., N. M. Rojas Hernández, V. Berovides Álvarez, E. Cruz Aldán, C. Chávez Hernández, J. A. Moguel Acuña, M. E. Pérez Escobar y M. G. Palacios Mendoza. 2009. Primer registro de Probstmayria tapiri (Nematoda: Atractidae) en Tapyirus bairdii (Gill, 1865) de la Sierra Madre del estado de Chiapas, México. Acta Zool. Mex. (n. s.) 25(1):83-91.

RESUMEN. Primer registro de Probstmayria tapiri en México y para la especie de Tapirus bairdii (tapir Centroamericano), en la que se hace una redescripción de este nematodo. Se encontró en 60 (41.09\%) muestras de excretas frescas recolectadas en las Reservas de la Biosfera "La Sepultura" y "El Triunfo" en la Sierra Madre de Chiapas, México. Las características morfológicas de los especimenes observados son nematodos pequeños con cutícula finamente estriada, fusiforme que gradualmente disminuye su diámetro hacia ambos extremos, terminando en una cola aguda. Boca hexagonal con tres labios bilobulados, faringe alargada, esófago con tres porciones: anterior, larga y delgada, media, corta y estrecha, piriforme, con un bulbo. El poro excretor se encuentra situado cerca del bulbo esofágico y ventral a la superficie del cuerpo. La vulva de la hembra esta cerca de la porción media del cuerpo. Las espículas del macho son asimétricas y curvadas. En la superficie ventral de la cola hay nueve papilas postanales. Estos resultados nos permiten ampliar la distribución geográfica del nematodo a Centroamérica y otra especie de tapir (T. bairdii). Finalmente enfatizamos que las condiciones ambientales de la Sierra Madre son óptimas para el desarrollo biológico de este nematodo de los miembros de la Familia Tapiridae en Centroamérica y Sudamérica.

Palabras clave: Reservas de la Biosfera, Probstmayria tapiri, Tapirus bairdii, Nematoda, Comensal, Sierra Madre de Chiapas. 
Güiris Andrade, D. M., N. M. Rojas Hernández, V. Berovides Álvarez, E. Cruz Aldán, C. Chávez Hernández, J. A. Moguel Acuña, M. E. Pérez Escobar \& M. G. Palacios Mendoza. 2009. First record of Probstmayria tapiri (Nematoda: Atractidae) in Tapyirus bairdii (Gill, 1865) from the Chiapas Sierra Madre, Mexico. Acta Zool. Mex. (n. s.) 25(1):83-91.

ABSTRACT. This is a first record of Probstmayria tapiri in Mexico, was found in 60 (41.09\%) fecal samples from Baird's tapir (Tapirus bairdii) in the Biosphera Reserves "La Sepultura" and "El Triunfo" in the Sierra Madre de Chiapas, Mexico. Morphological characteristics of small nematode spindle-shaped forms truncated anteriorly and with a long, gradually attenuated, acutely pointed tail. Mouth with tree lips, pharynx elongated, aesophagus with tree portions: Anterior portion, long and slender, posterior portion, shorter, and pyriform portion, with a bulb. The excretory pore is situated near oesophagi bulb and ventral surface of the body. Vulva of female at about the middle of the body. Spicules assymetrical and curved. On the ventral surface of the tail there are nine small postanal papillae. These results allow us to extend their geographic distribution of nematode to Central America and another species of Tapir's (T. bairdii). Finally we emphasized that the environmental conditions of La Sierra Madre de Chiapas, are optimal for the biological development of this nematode from tapirs of Central and South America

Keys word: Reserves of the Biosphere, Probstmayria tapiri, Tapirus bairdii, Nematoda, Commensal, Sierra Madre de Chiapas.

\section{INTRODUCCIÓN}

El género Probstmayria fue descrito por Probstmayr (1865) bajo el nombre de Oxyuris vivipara encontrado en ciegos de caballos domésticos. Posteriormente, Perroncito (1882) y Fiorentini (1890) lo registran en Italia; Jerke (1902) de Alemania, lo observó en caballos; Looss (1902) en burros de Egipto y Ranson (1907) en caballos domésticos de Estados Unidos de Norteamérica. Dicho género incluye especies reportadas para otros grupos de vertebrados como Suidos (jabalí africano), Antropoides (chimpancé, gorila), Chelonia (tortuga terrestre sudafricana) en Asia y África.

El primer reporte en fauna silvestre para el neotrópico americano se observó en el ciego de un tapir sudamericano (Tapirus terrestris) en la Guyana Francesa (Van Waerebeke et al. 1988). Estos autores describen y nombran a esta nueva especie como Probstmayria tapiri.

El presente trabajo informa la primera identificación de Probstmayria tapiri en México y para la especie de Tapirus bairdii (tapir centroamericano).

\section{MATERIAL Y MÉTODO}

El material estudiado proviene de excretas frescas de Tapirus bairdii con menos de 48 horas de deposición, (caracterizadas por su textura, olor penetrante, color verde oscuro brillante, conformación, humedad, evitando las que presentaron desarrollo de hongos y semillas) recolectadas del suelo, arroyos, letrinas y posas entre altitudes que comprenden los 1040 y $1545 \mathrm{~m} \mathrm{~s} \mathrm{n} \mathrm{m} \mathrm{en} \mathrm{las} \mathrm{zonas} \mathrm{núcleo} \mathrm{de} \mathrm{las} \mathrm{Reservas} \mathrm{de} \mathrm{la}$ Biosfera "La Sepultura" (Polígono La Palmita con 1,937.675 ha de superficie) y "El Triunfo" (Polígono I con 11,549.75 ha de superficie ) ubicadas entre las coordenadas geográficas $16^{\circ} 21^{\prime} 56^{\prime \prime}$ y $16^{\circ} 20^{\prime} 18^{\prime \prime}$ de latitud norte y $93^{\circ} 58^{\prime} 31^{\prime \prime}$ y $93^{\circ} 53^{\prime} 10^{\prime \prime}$ de 
longitud oeste (PEOT, 2004) en la Sierra Madre de Chiapas, México (Fig. 1); durante el periodo de febrero del 2002 a febrero del 2005 (INE-SEMARNAT, 1999 a,b). El método de recolecta utilizado fue por medio de un trayecto en línea de amplitud y longitud variable con recorridos a pie, debido a las condiciones de vegetación y topografía del lugar (Burnham et al. 1980; Clemente 1996).

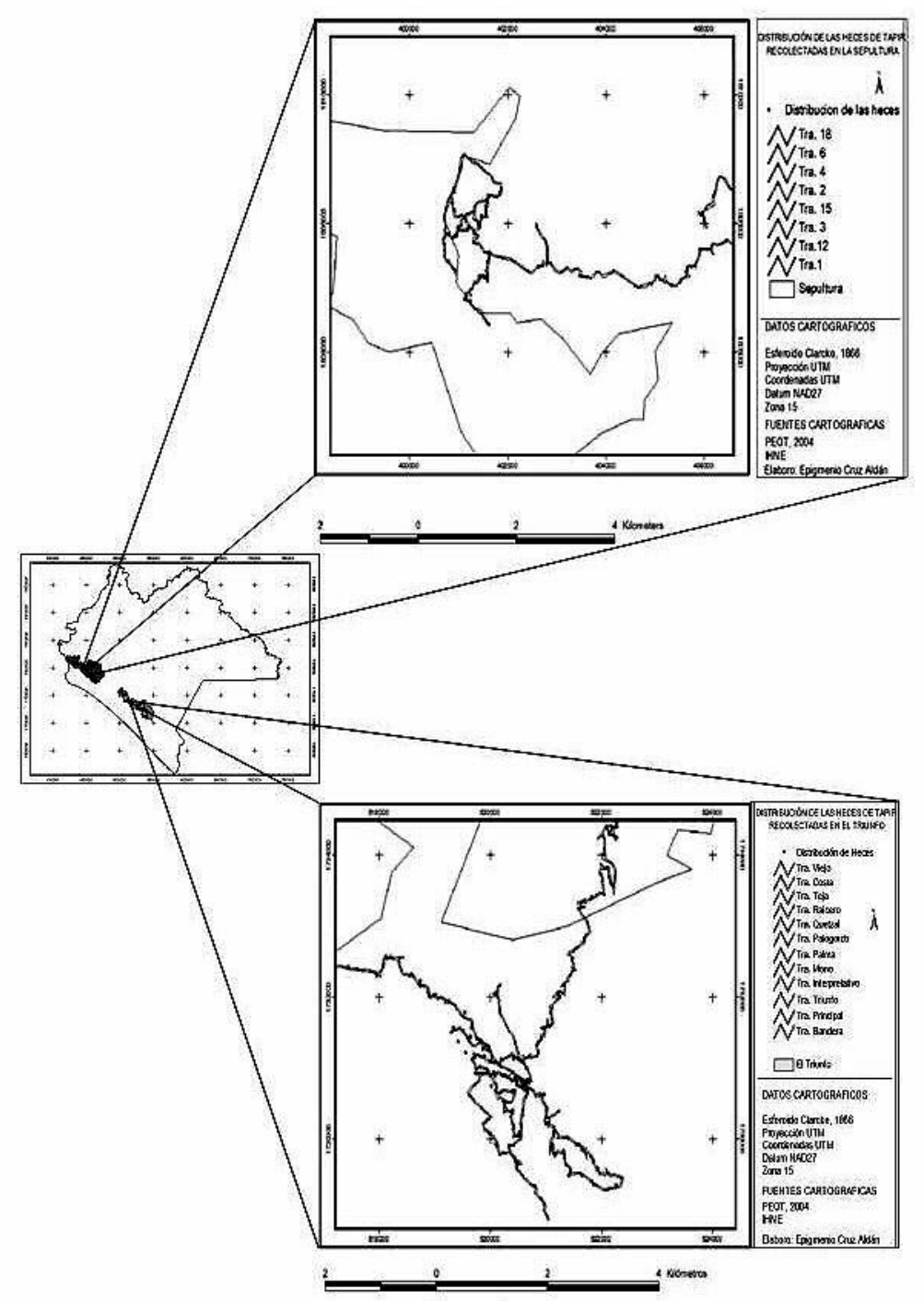

Figura 1. Ubicación geográfica y distribución de excretas positivas a Probstmayria tapiri en trayectos de las Reservas de la Biósfera "La Sepultura" y "El Triunfo" Chiapas, México. 
Güiris et al.: Probstmayria tapiri en Tapirus bairdii de Chiapas, México

Cuadro 1. Descripción morfométrica comparativa de Probstmayria tapiri según Van Waerebeke et al. (1988), con especimenes (hembras $n=10$, macho $n=1$ ) obtenidos de excretas de Tapirus bairdii en Reservas de la Biosfera de la Sierra Madre de Chiapas, México.

\begin{tabular}{|c|c|c|}
\hline \multirow[t]{2}{*}{ DESCRIPCION MORFOLOGICA } & \multicolumn{2}{|c|}{ MEDIDAS Probstmayria tapiri $(\mu \mathrm{m})$} \\
\hline & Espécimen de T. bairdii & Espécimen de T. terrestris (VW) \\
\hline Longitud total: $\mathrm{M}$ & $1,742.0$ & $1,530.0-1640.0$ \\
\hline Longitud total: $\mathrm{H}$ & $2,115.1(2,011.7-2,218.5)$ & $1,750(1,670-1,920.0)$ \\
\hline Ancho: M & 88.4 & $66.0-84.0$ \\
\hline Ancho: $\mathrm{H}$ & $91.0(87.1-94.9)$ & $94.0(76.0-111.0)$ \\
\hline Longitud esófago: $\mathrm{M}$ & 315.1 & $293.0-306.0$ \\
\hline Longitud esófago: $\mathrm{H}$ & $317.0(311.1-323.0)$ & $314.0(295.0-334.0)$ \\
\hline Distancia anillo nervioso al ápice: $\mathrm{M}$ & 168.6 & $143.0-155.0$ \\
\hline Distancia anillo nervioso al ápice: $\mathrm{H}$ & $173.1(169.0-177.3)$ & $154.0(139.0-167.0)$ \\
\hline Distancia poro excretor al ápice: $\mathrm{M}$ & 272.1 & $235.0-251.0$ \\
\hline Distancia poro excretor al ápice: $\mathrm{H}$ & $274.3(270.2-278.4)$ & $259.0(229.0-291.0)$ \\
\hline Distancia vulva al ápice: $\mathrm{H}$ & $899.1(870.0-928.1)$ & $816.0(750.0-907.0)$ \\
\hline Distancia ano a punta de la cola: $\mathrm{M}$ & 576.2 & $477.0-542.0 *$ \\
\hline Distancia ano a punta de la cola: $\mathrm{H}$ & $656.1(641.4-670.7)$ & $566.0(481.0-602.0) *$ \\
\hline Longitud espícula derecha: M & 66.2 & $64.0(60.0-67.0)$ \\
\hline Longitud espícula izquierda: $\mathrm{M}$ & 41.5 & $43.0(41.0-48.0)$ \\
\hline Ancho gubérnaculos & 15.0 & \\
\hline Longitud total de larva II & $826.2-1,062.6$ & $1,000.0$ \\
\hline Ancho de larva II & $29.5-47.2$ & \\
\hline Diámetro anillo quitinoso bucal: $\mathrm{M}$ & 18.8 & \\
\hline Diámetro anillo quitinoso bucal: $\mathrm{H}$ & $17.4-20.3$ & \\
\hline Longitud faringe: $\mathrm{M}$ & 37.3 & \\
\hline Longitud faringe: $\mathrm{H}$ & $40.5(39.0-41.1)$ & \\
\hline Ancho faringe & $4.3-7.3$ & \\
\hline Ancho cuerpo del esófago: $M$ & 21.6 & \\
\hline Ancho cuerpo del esófago: $\mathrm{H}$ & $23.0(22.6-23.3)$ & \\
\hline Ancho istmo del esófago: M & 15.4 & \\
\hline Ancho istmo del esófago: $\mathrm{H}$ & $15.6(15.3-16.0)$ & \\
\hline Longitud bulbo del esófago: M & 55.2 & \\
\hline Longitud bulbo del esófago: $\mathrm{H}$ & $57.6(56.4-58.8)$ & \\
\hline Ancho bulbo del esófago: M & 42.2 & \\
\hline Ancho bulbo del esófago: $\mathrm{H}$ & $47.6(45.2-50.0)$ & \\
\hline Longitud Poro excretor: M & 35.6 & \\
\hline Longitud Poro excretor: $\mathrm{H}$ & $39.8(37.6-42.0)$ & \\
\hline Ancho Poro excretor: M & 29 & \\
\hline Ancho Poro excretor: $\mathrm{H}$ & $28.1(26.0-29.6)$ & \\
\hline Ancho de Intestino: $\mathrm{H}$ & A $47.2, \mathrm{Md} 36.2, \mathrm{P} 29.0$ & \\
\hline Longitud del útero & $294.6-356.7$ & \\
\hline Ancho del útero & $59.4-65.6$ & \\
\hline Distancia entre ano y ápice: $\mathrm{H}$ & $1,103.3$ & \\
\hline Distancia entre ano y vulva: $\mathrm{H}$ & $289.1-312.7$ & \\
\hline
\end{tabular}

VW.- Van Waerebeke et al. (1988). $\mu$ m.- Micrómetro. M.- Macho. H.- Hembra. A.- Ancho. Md.- Medio. P.- Posterior. * Longitud de cola. 
Las excretas se conservaron en formaldehído al 10\% (1 volumen de formol por 4 volúmenes de excreta) y se procesaron mediante la técnica de sedimentación y micrometría (Thienpont et al. 1979). El material recolectado se identificó en el Laboratorio de la Policlínica y Diagnóstico Veterinario (autorizado por la Secretaría de Sanidad Animal, SAGARPA No. 162) en Tuxtla Gutiérrez, Chiapas, México, y la Facultad de Biología de la Universidad de la Habana, Cuba; siguiendo la nomenclatura y sistemática para la identificación de Taxa mediante claves estándar (Ransom, 1907, Van Waerebeke et al. 1988). Los especímenes y fototipos se depositaron en la Colección Biológica: "Agentes Parasíticos y Endosymbiontes / Endocomensales en Fauna del Estado de Chiapas, México", del Cuerpo Académico: Estudios Biomédicos y de Sanidad en Fauna. Facultad de Medicina Veterinaria y Zootecnia de la Universidad Autónoma de Chiapas y Facultad de Biología de la Universidad de la Habana, Departamento de Microbiología. Registro: UCH$\mathrm{Tb} / \mathrm{N} / \mathrm{K} / \mathrm{Pt} 01$.

\section{RESULTADOS}

Hábitat, Tipo de Huésped y Localidad: Intestino (excretas) de Tapirus bairdii, en Reservas de la Biosfera "La Sepultura" (Polígono "La Palmita") y "El Triunfo" (Polígono I), en la Sierra Madre de Chiapas, México.

Prevalencia: 60 muestras positivas de 146, 41.09\%

Redescripción de Probstmayria tapiri obtenido de excretas en Tapirus bairdii (Cuadro 1).

Macho. Dimensiones y Estructuras: Nematodo adulto pequeño con cutícula finamente estriada más gruesa en el borde dorsal en comparación con el ventral, fusiforme que gradualmente disminuye su diámetro hacia ambos extremos, terminando en una cola aguda. Longitud total 1,742.0 $\mu \mathrm{m}$ fusiformes. Ancho 88.4 $\mu \mathrm{m}$ que disminuye gradualmente su diámetro hacia ambos extremos. Boca hexagonal rodeada por un borde que forman los tres labios bilobulados, con un diámetro de 18.8 $\mu \mathrm{m}$ compuesto por seis papilas labiales. Faringe cilíndrica y elongada con una longitud de $37.3 \mu \mathrm{m}$ (Fig. 2B). Esófago con una longitud de $315.1 \mu \mathrm{m}$ y $21.6 \mu \mathrm{m}$ de ancho, compuesto de un cuerpo cilíndrico, un istmo corto con un ancho de $15.4 \mu \mathrm{m}$ y un bulbo bien definido con una longitud de $55.2 \mu \mathrm{m}$ y un ancho de $42.2 \mu \mathrm{m}$. Anillo nervioso localizado en el segundo tercio del esófago cerca de la unión con el tercio posterior, a una distancia del ápice de $168.6 \mu \mathrm{m}$. Poro excretor dentro de una vesícula de paredes quitinosas en la unión del bulbo e istmo del esófago, a una distancia del ápice de $272.1 \mu \mathrm{m}$, la longitud y el ancho es de 35.6 y $29.0 \mu \mathrm{m}$ respectivamente (Fig. 2A). Intestino ancho uniforme adelgazándose ligeramente hacia el ano, con un lumen estrecho. En el extremo caudal hay 9 papilas cloacales pequeñas, Gubernáculos asimétricos y pequeños. Espícula izquierda en forma de gancho con una longitud de 


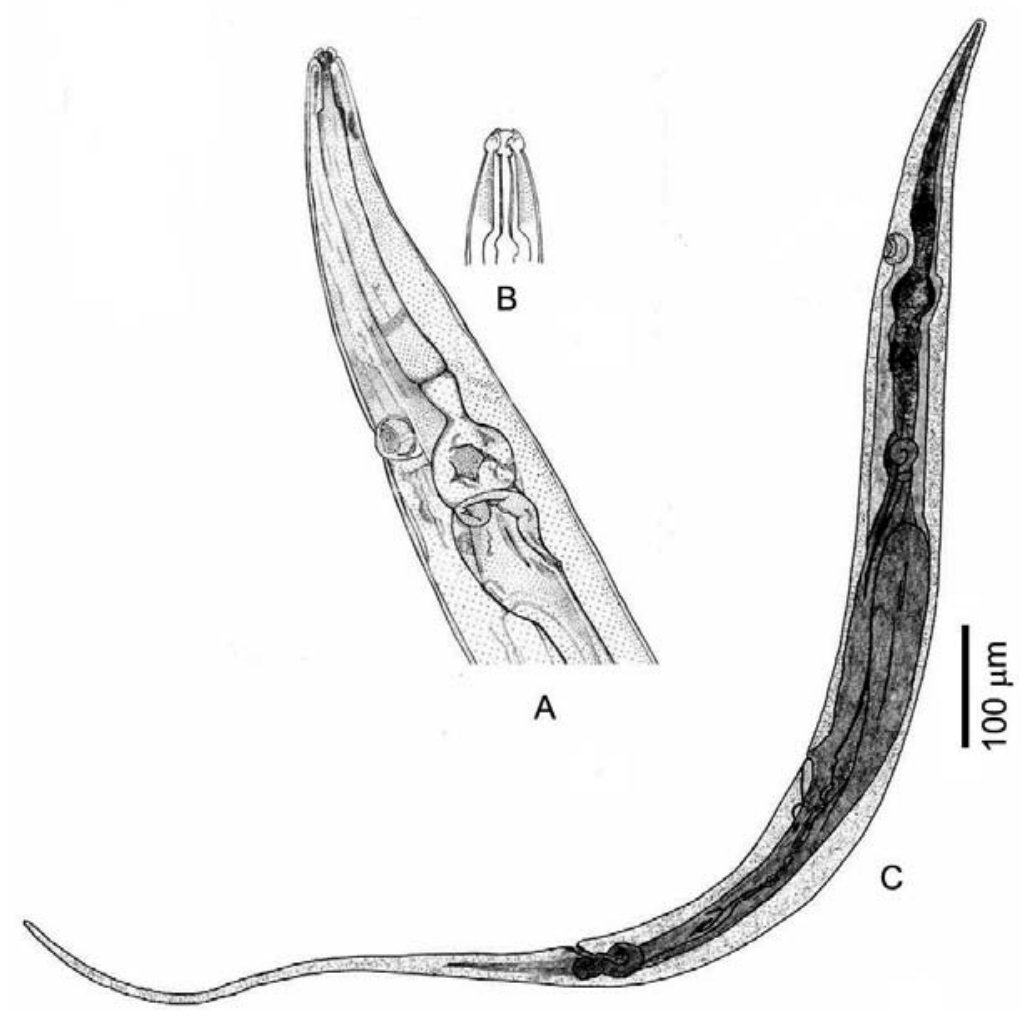

Figura 2. Espécimen adulto de Probstmayria tapiri: (A) extremo anterior donde se observa el poro excretor y el esófago largo compuesto de un cuerpo cilíndrico, un istmo corto y un bulbo bien definido. (B) extremo cefálico, mostrando la faringe. (C) hembra adulta grávida con larva en el tercio medio.

$66.2 \mu \mathrm{m}$ y un ancho en la base de $15.0 \mu \mathrm{m}$, la espícula derecha es más corta que la izquierda con una longitud de $41.5 \mu \mathrm{m}$ y un ancho en la base de $15.0 \mu \mathrm{m}$. Cola larga cilíndrica la cual va adelgazándose terminando abruptamente en punta (no filiforme), la distancia entre el ano y la punta de la cola es de $576.2 \mu \mathrm{m}$.

Hembra. Dimensiones y Estructuras: Nematodos adultos de mayor tamaño que el macho, con cutícula y forma similares a la descrita en el nematodo macho. Longitud total 2,115.1 $\mu \mathrm{m}$. Ancho $91.0 \mu \mathrm{m}$ que disminuye gradualmente su diámetro hacia ambos extremos. Boca hexagonal rodeada por un borde que forman los tres labios bilobulados, con un diámetro de $17.4-20.3 \mu \mathrm{m}$ y $8.7-10.1 \mu \mathrm{m}$ de ancho, compuesto por seis papilas labiales. Faringe cilíndrica y elongada con una longitud de $40.5 \mu \mathrm{m}$ con un diámetro de $4.3-7.3 \mu \mathrm{m}$. Esófago con una longitud de $317.0 \mu \mathrm{m}$ 
y $23.0 \mu \mathrm{m}$ de ancho, compuesto de un cuerpo cilíndrico, un istmo corto con un ancho de $15.6 \mu \mathrm{m}$ y un bulbo bien definido con una longitud de $57.6 \mu \mathrm{m}$ y un ancho de 47.6 $\mu \mathrm{m}$. Poseen un anillo nervioso similar al descrito en el macho, a una distancia del ápice de $173.1 \mu \mathrm{m}$. El poro excretor, es similar a lo descrito en el macho, a una distancia del ápice de $274.3 \mu \mathrm{m}$, la longitud y el ancho es de 39.8 y $28.1 \mu \mathrm{m}$ respectivamente. El intestino tiene un ancho uniforme adelgazándose ligeramente hacia el ano, con un lumen estrecho, el ancho intestinal en la parte posterior al esófago es de $47.2 \mu \mathrm{m}$ en la parte media anterior al útero $36.2 \mu \mathrm{m}$ y en la parte posterior cerca de las glándulas del recto y ano $29.0 \mu \mathrm{m}$. Recto estrecho rodeado de glándulas. Útero ubicado en la parte media corporal y el tamaño depende del estado de desarrollo de larva en su interior y este se extiende desde un punto de unión con la vagina en dirección anterior, la longitud es de 294.6 - $356.7 \mu \mathrm{m}$ y el ancho de 59.4 - $65.6 \mu \mathrm{m}$ (Fig. 2C). Vulva en posición preecuatorial. Distancia entre el ano y la vulva es de $289.1-312.7 \mu \mathrm{m}$. Cola similar a la descrita en macho, la distancia entre el ano y la punta de la cola es de $656.1 \mu \mathrm{m}$

Larva. El aspecto morfológico observado es similar al de hembra adulta. La longitud de la larva II es de $826.2-1,062.6 \mu \mathrm{m}$ y el ancho de $29.5-47.2 \mu \mathrm{m}$. En el presente estudio observamos que la cola está cubierta por una vaina gruesa y bien diferenciada. La distancia de la punta de cola y el extremo de la vaina es de 53.6 $65.2 \mu \mathrm{m}$, este aspecto no se había descrito anteriormente.

\section{DISCUSIÓN}

En estudios de nematodosis en tapiridos, se reportan las siguientes familias: Trichostrongylidae, Ancylostomatidae y Strongylidae (Durette-Desset et al. 1997, Cruz et al. 2006), que difieren en cuanto al hallazgo de nematodos comensales (Probstmayria tapiri) de la familia Kathlaniidae en este estudio y a lo reportado por Van Waerebeke et al. (1988). Previo a este estudio, Probstmayria tapiri únicamente ha sido reportado en Tapirus terrestris en Sudamérica por Van Waerebeke et al. (1988), por lo que la presente investigación amplía su distribución geográfica a Centroamérica y otra especie de tapir (Tapirus bairdii), lo que permite conocer el endemismo de este nemátodo en otras regiones del neotrópico.

La prevalencia de Probstmayria tapiri, es moderadamente baja (41.09\%). La escasa frecuencia de hallazgos de este nematodo en tapires de otras regiones de Centroamérica y Sudamérica se debe a la dificultad para disponer de excretas frescas. Así como, al desconocimiento morfológico de este nematodo y a la falta de implementación de técnicas de diagnóstico en los proyectos de investigación biomédica sobre endocomensales, endosimbiontes y otros agentes parasíticos (Güiris y Cruz, 2003, IUCN/TSG, 2005).

El género Probstmayria representa el único ejemplo de multiplicación de un nematodo en el cuerpo de un huésped, no se considera un parásito, pues aunque viven 
y se alimentan del contenido intestinal, no tienen ningún efecto patógeno sobre el huésped (Lapage 1956, 1968, Dunn 1978), por lo que se debe considerar como un agente comensal miembro de la microbiota intestinal de Tapirus bairdii, ya que los hallazgos de Probstmayria tapiri en excretas frescas, concuerda con lo descrito por Ranson (1907) para Probstmayria vivipara en équidos de los Estados Unidos de América, donde menciona la deposición de los nematodos en las excretas y la factibilidad para permanecer vivos cuatro a cinco días después de la deposición. En contraste con Van Waerebeke et al. (1988), se encontró una baja proporción de machos con relación al número de hembras. Los especimenes adultos y larva II de Probstmayria tapiri recobrados en excretas de Tapirus bairdii, fueron en morfología y micrometría, similares a la descripción previa realizada por Van Waerebeke et al. (1988) en Tapirus terrestris. Adicional a la descripción original: la cutícula estriada es más gruesa en el borde dorsal en comparación al borde ventral, la cola es gruesa larga y cilíndrica que va adelgazándose terminando abruptamente en punta (no filiforme), y se describen nuevos registros de dimensiones en el nematodo adulto: anillo quitinoso bucal, longitud de faringe, ancho del esófago, longitud y ancho del poro excretor, longitud y ancho del poro excretor de Probstmayria tapiri. Finalmente enfatizamos que las condiciones ambientales de la Sierra Madre de Chiapas y las de la región de Regina en la Guyana Francesa (Van Waerebeke et al. 1988) son óptimas para el desarrollo biológico de este nematodo comensal de los miembros de la Familia Tapiridae en Centroamérica y Sudamérica.

AGRADECIMIENTOS: Agradecemos el apoyo brindado por el personal de las reservas de la biosfera El Triunfo y La Sepultura (de manera especial a los guarda parques de El Triunfo). Agradecemos al Ilustrador científico José Uriostegui su valiosa colaboración y autoría de las figuras. Se agradece al Instituto de Historia Natural y Ecología, Proyecto Mamíferos Chiapanecos en Peligro, por el apoyo brindado. Así como al Proyecto "Estudios Biomédicos y de Sanidad en Fauna Silvestre, Cautiva y Nativa del Estado de Chiapas" del Cuerpo Académico: Estudios Biomédicos y de Sanidad en Fauna" de la Facultad de Medicina Veterinaria y Zootecnia de la Universidad Autónoma de Chiapas.

\section{LITERATURA CITADA}

Burnham, K.P., Anderson, D.R. \& J.L. Laake. 1980. Estimation of density from line transect sampling of biological populations. Wildlife Monograph, 72: 202.

Clemente, F. 1996. Manual teórico práctico de métodos de estimación de tamaños de población de fauna silvestre. Colegio de postgraduados. Estado de México, México.

Cruz A.E, Lira T.I, Güiris A.D.M, Osorio S.D. \& Quintero M. Ma.T. 2006. Parásitos del tapir Centroamericano Tapirus bairdii (Perissodactyla: Tapiridae) en Chiapas, México. Revista de Biologia Tropical. (Int. J. Trop. Biol.). 54 (2): 445 - 450.

Dunn, A.M. 1978. Helmintología Veterinaria. $2^{\text {a }}$ edición. Editorial Moderno, S.A. de C.V., México, D.F.

Durette-Desset M.C., Chabaut A.G. \& Sutton C.A. 1997. Tapironema coronatum n. gen., n. sp. (Trichostrongyloidea - Cooperiidae - Obeliscoidinae), A Parasite of Holochilus brasiliensis and Tapirus terrestris. Parasite. 4: 227-232. 
Fiorentini, A. 1890. Sull'ossiuride vivipara (Oxyuris vivipara Probsmayr) cenni descrittivi. Bulletin Scientific of Pavia, 12(1: 21-25.

Güiris A.D.M. \& Cruz A.E. 2003. Estudios Biomédicos y de Sanidad en Tapirus bairdii del Estado de Chiapas, México. En: Estudios Biomédicos y de Sanidad en Fauna Silvestre Nativa y Cautiva del Estado de Chiapas. Proyecto de Investigación continúa desde 1988, a la fecha. Policlínica y Diagnóstico Veterinario, Instituto de Historia Natural y Ecología (ZooMAT) Gobierno del Estado de Chiapas (IHNE), Universidad Autónoma de Chiapas, Investigación y Posgrado. Tuxtla Gutiérrez Chiapas.

INE-SEMARNAP. 1999a. Programa de manejo de la Reserva de la Biosfera El Triunfo, México. Instituto Nacional de Ecología - Secretaria de Medio Ambiente Recursos Naturales y Pesca. México, D.F.

INE-SEMARNAP. 1999b. Programa de manejo Reserva de la Biosfera La Sepultura. Chiapas, México. Instituto Nacional de Ecología - Secretaría de Medio Ambiente Recursos Naturales y Pesca. México, D.F.

IUCN/TSG. 2005. PHVA DANTA CENTROAMERICANA Tapirus bairdii. BRIEFING BOOK. Baird's Tapir (Tapirus bairdii) Conservation Workshop Population and Habitat Viability Assessment (PHVA). Section 4: Baird's Tapir Ecology. Section 6: Veterinary Aspects in Baird's Tapir. Tapir Specialist Group (TSG), Conservation Breeding Specialist Group (CBSG), The Belize Zoo y Tropical Education Center (TEC), Belize, Central America, Houston Zoo Inc. United States, American Zoo and Aquarium Association (AZA), Tapir Taxon Advisory Group (TAG), European Association of Zoos and Aquaria (EAZA). Apple valley, MN, USA.

Jerke, H.W.M. 1902. Eine parasitische Anguillula des Pierdes. Archiv fur Wissenschaftliche und Praktische Thierheilkunde, Berl., 29(1-2): 113-127.

Lapage, G. 1956. Monnig's Veterinary Helminthology and Entomology. Fourth edition. The Williams and Wilkins Company. Baltimore.

Lapage, G. 1968. Parasitología Veterinaria. Editorial Continental, S.A. de C.V., México, D.F.

Looss, A. 1902. The Sclerostomidae of horses and donkeys in Egypt. Records of the Egyptian Government. School of Medicine. Cairo 25-139.

Perroncito, E. 1882. I parassiti dell uomo e degli animali utili. Delle più comuni malattie da essi prodotte profilassi e cura relativa. Milano. 12: 233, 506.

Probstmayr, W. 1865. Oxyuris vivipara. Wschnschr. F. Tierheilkd und Viehzicht Augsburg, 9(23):178180.

PEOT. 2004. Programa de Ordenamiento Territorial del Estado de Chiapas. Gobierno del Estado.

Ransom, B.H. 1907. Probstmayria vivipara (Probstmayr, 1865) Ransom 1907, a Nematode of Horses Heretofore Unreported from the United States. Transactions of the American Microscopical Society, Twenty-Eighth Annual Meeting. (march) 27: 33-40.

Thienpont, D., Rochette, F. \& Vanparij, O.F. 1979. Diagnóstico de las helmintiasis por medio del examen coprológico. Jenssen Reasearch Foundation, Beerse, Bélgica.

Van Waerebeke, D., Chabaud, A.G. et Anthony, G. 1988. Probstmayria tapiri n. sp, Nematode parasite d'un Tapir du Nouveae Monde. Bulletin Museum National History Natural, Paris, 4a. sér., 10. section A, 1: 3-8.

Recibido: 26 de noviembre de 2007

Aceptado: 5 de septiembre de 2008 